\section{Cemento-Osseous Dysplasias: Imaging Features Based on Cone Beam Computed Tomography Scans}

Paulo Henrique Pereira Cavalcanti, Eduarda Helena Leandro Nascimento, Maria Luiza dos Anjos Pontual, Andréa dos Anjos Pontual, Priscylla Gonçalves Correia Leite de Marcelos, Danyel Elias da Cruz Perez, Flávia Maria de Moraes Ramos-Perez
Department of Clinical and Preventive Dentistry, UFPE - Universidade Federal de Pernambuco, Recife, PE, Brazil

Correspondence: Eduarda Helena Leandro Nascimento, Avenida Limeira, 901, 13414-903 Piracicaba, SP, Brazil. Tel: +55-19-8294-6677. e-mail: eduarda.hln@gmail.com
Imaging exams have important role in diagnosis of cemento-osseous dysplasia (COD). Cone beam computed tomography (CBCT) stands out for allowing three-dimensional image evaluation. This study aimed to assess the prevalence of cases diagnosed as COD on CBCT scans, as well identify the main imaging features related to these lesions. An analysis was performed in a database containing 22,400 radiological reports, in which all cases showing some type of COD were initially selected. These CBCT exams were reevaluated to confirm the radiographic diagnosis and determine the prevalence and distribution of the types of COD with regard to gender, age and preferred location, while describing its most common imaging aspects. Data were presented using descriptive analyses. There were 82 cases diagnosed as COD in the CBCT images (prevalence of $0.4 \%$ ). The distribution of patients was $11(13.4 \%)$ male and $71(86.6 \%)$ female, with a mean age of 49.8 years (age-range 17-85 years). There were 47 (57.3\%) cases of periapical COD, 23 (28\%) of focal COD and $12(14.6 \%)$ of florid COD. The mandible was more affected than the maxilla. In most cases, the lesions were mixed or hyperdense. All COD had well-defined limits and there were no cases of tooth displacement. In conclusion, periapical COD was the most common type and the most affected bone was the mandible. Imaging evaluation is critical for diagnosis and dentists should bear in mind all possible radiographic presentations of COD in order to prevent misleading diagnoses and consequently, inadequate treatments.

\author{
Key Words: cemento-osseous \\ dysplasia, cone-beam \\ computed tomography, florid \\ cemento-osseous dysplasia, \\ focal cemento-osseous \\ dysplasia, periapical cemento- \\ osseous dysplasia.
}

\section{Introduction}

Cemento-osseous dysplasia (COD) is a benign disorder belonging to a pathological group known as fibro-osseous lesions. Although it is a widely used term, 'fibro-osseous lesion' does not represent a specific diagnosis, but a similar biological process in some diseases, in which the normal bone is replaced by fibrous tissue containing foci of mineralized substance. In COD, the mineral content may be formed of bone, cement or both. Although its origin is not yet fully understood, it is believed that the CODs are derived from periodontal ligament tissue (1).

The current classification proposed by the World Health Organization (WHO) (2) subdivides the COD into 3 groups, according to their location: periapical COD, if it occurs in the periapical region of the anterior teeth; focal COD, if it is associated with a single tooth, and florid COD, when there is multifocal involvement, occurring in more than one jaw quadrant. These three divisions are merely variants of the same pathological process (1). The lesions predominantly affect women, black patients, with peak incidence during the fourth and fifth decades of life (2-4).

COD are usually asymptomatic and located in the periapical region of teeth with pulp vitality and are commonly identified in routine radiologic exams $(1,3,5)$. Its imaging aspects vary according to the stage of development of the lesion. In the osteolytic or early stage, there is resorption of bone tissue and deposition of wellvascularized connective tissue, therefore, radiographically the COD appears radiolucent or hypodense. Due to progressive deposition of mineral content within the lesion, the intermediate and mature stages show mixed and radiopaque/hyperdense images respectively $(1,3,5,6)$. Depending on the stage of COD, the list of differential diagnoses may be extensive, ranging from cystic or inflammatory periodontal lesions (6-9) through even some odontogenic tumors (4). Thus, the dentist must be careful to prevent misdiagnosis and inadequate procedures (treatment or endodontic re-treatment, apicectomy and exodontia), since the COD in most cases are self-limiting lesions and require only radiographic follow-up $(1,5,9,10)$.

The diagnosis of COD is usually made by correlation of demographic information (age, gender and ethnicity), clinical (location and number of lesions) and imaging (image type and density) features (11). The diagnosis based on these features is essential because the affected bone tissue is more susceptible to infections and invasive 
procedures such as biopsies could cause this effect (12). In this sense, cone beam computed tomography (CBCT) has been shown a valuable tool for identifying these lesions, as it allows accurate evaluation of bone tissue. Moreover, CBCT is increasingly used in Dentistry, allowing access to these images by professionals in the most varied areas, who must be able to recognize these conditions and properly manage the patient.

Therefore, the aim of this study was to evaluate the distribution of cases diagnosed as cemento-osseous dysplasia in CBCT scans as regards gender, age and preferred location. In addition, we described the most common imaging features related to COD types by using CBCT and comparing them with the literature.

\section{Material and Methods}

Approval for the present study was granted by the Local Research Ethics Committee, Federal University of Pernambuco, Brazil (protocol \#02588812.1.0000.5208).

A total of 22,400 radiological reports from a private radiology clinic obtained over a five years' period were retrospectively examined. For this purpose, a search was conducted in this database with the key words "dysplasia",

"focal", "florid" and "periapical cemental", resulting in 300 CBCT scans being found. These images were carefully reevaluated to compose the final sample of this study, in order to exclude cases diagnosed as fibrous dysplasia or other types of conditions other than COD. Inclusion criteria were the presence of periapical, focal or florid osseous dysplasia located in the maxilla and/or mandible; and CBCT images of a quality suitable for diagnosis.

All scans were acquired by cone beam CT scanners i-CAT classic and i-CAT next generation (Xoran Technologies, Ann Arbor, $\mathrm{MI}$ and Imaging Sciences International, Hatfield, $\mathrm{PA}, \mathrm{USA})$, operating at $120 \mathrm{kVp} / 8 \mathrm{~mA}$, voxel size ranging from $0.2 \mathrm{~mm}-0.4 \mathrm{~mm}$, acquisition time of 20 to $26 \mathrm{~s}$ and a FOV covering entire jaws. Images were reconstructed using the XORAN software program (Xoran Technologies) and evaluated simultaneously by two oral radiologists with experience in analyzing tomographic images, on a computer monitor (19-inch LCD monitor, Toshiba America Information System, Inc., China), under dim lighting conditions. The use of "zoom" tool and manipulation of brightness and contrast was allowed.

The information on demographic data (age and gender) were obtained from the radiological reports. The types of COD and their imaging features (region involved; affected tooth; lesion density; presence of hypodense halo; margin type; expansion; thinning or perforation of the cortical bone; root resorption; tooth displacement; and presence of endodontic treatment of the affected teeth) were analyzed in CBCT images. In cases of COD related to endodontically treated teeth with root resorption, this resorption was not recorded due to uncertainty about the origin of resorption being inflammatory or having been caused by dysplasia. To classify the type of osseous dysplasia, the criteria proposed by WHO (2) were used, in which CODs have been divided into the following groups: periapical COD, focal COD and florid COD, according to their clinical-imaginological characteristics.

In case of disagreement between the examiners, a third examiner, an oral radiologist with more than 15 years of experience, was consulted to reach a final diagnosis. The data collected were tabulated and descriptive statistical analyses were performed using Microsoft Excel Software (Microsoft Office Excel 2007, Microsoft, Redmond, WA, USA).

\section{Results}

Out of a total of 22,400 radiological reports, CODs were found and re-examined in 82 CBCT scans (prevalence of $0.4 \%)$, whose distribution was $11(13.4 \%)$ males and 71 (86.6\%) females, aged between 17 and 85 years (mean \pm SD: $49.8 \pm 13.3$ years).

Examples of CBCT images of periapical and focal COD are shown in Figures 1 and 2, respectively. Female were more affected than male in all types of COD. There was a higher prevalence of periapical COD (57.3\% of cases); followed by focal COD (28\%) and florid COD (14.6\%). The age of patients with focal COD was on average a decade below of those with periapical and florid COD, although the age that they appeared most often had been similar for all COD types (modes values of 52, 51 and 58 for periapical, focal and florid COD, respectively). Distribution of the demographic features is summarized in Table 1.

Table 2 shows the data referring to the clinical and imaging features for each COD group. Irrespective of COD type, the mandible was the most affected bone and the main aspect of the lesions was hyperdensity surrounded

Table 1. Distribution of the demographic features of the types of cemento-osseous dysplasia

\begin{tabular}{lccc}
\hline \multirow{2}{*}{ Demographic data } & \multicolumn{3}{c}{ Cemento-osseous dysplasia } \\
\cline { 2 - 4 } & Periapical & Focal & Florid \\
\hline Total $(\mathrm{n}=82)$ & $47(57.3 \%)$ & $23(28 \%)$ & $12(14.6 \%)$ \\
Sex & & & \\
Male $(\mathrm{n}=11)$ & $6(12.8 \%)$ & $4(17.4 \%)$ & $1(8.3 \%)$ \\
Female $(\mathrm{n}=71)$ & $41(87.2 \%)$ & $19(82.6 \%)$ & $11(91.7 \%)$ \\
Age (years) & & & \\
Mean $\pm \mathrm{SD}$ & $52.3 \pm 12.9$ & $42.1 \pm 13.8$ & $54.4 \pm 7.4$ \\
Range & $28-85$ & $17-63$ & $37-62$ \\
\hline
\end{tabular}


by a hypodense rim. In all CBCT scans analyzed, the margin of the lesion was well-defined and there were no cases of tooth displacement related to COD.

As regards the effects on adjacent structures, the florid COD was more related to perforation and expansion of cortical bone and root resorption, while the periapical COD was associated with cortical thinning in more than half of the cases. Considering the presence of endodontically treated teeth, there was a higher prevalence in cases of focal and florid COD (Table 2).

The present study also evaluated which teeth were affected by the lesions. For this analysis, only the focal and periapical CODs were considered, because florid COD typically affects multiple regions. Figure 3 shows the distribution of teeth involved with the COD. The teeth most affected by periapical COD were the mandibular central incisors, representing 48.1\% (65 teeth) of the 135 anterior teeth involved. In focal COD, the most affected teeth were the right mandibular first premolar and second molar, with prevalence of 21.7\% (5 teeth) and 17.4\% (4 teeth), respectively.

\section{Discussion}

This study evaluated the prevalence of diagnosis and imaging feature of COD by means of CBCT. This approach is important because, so far, little information has been
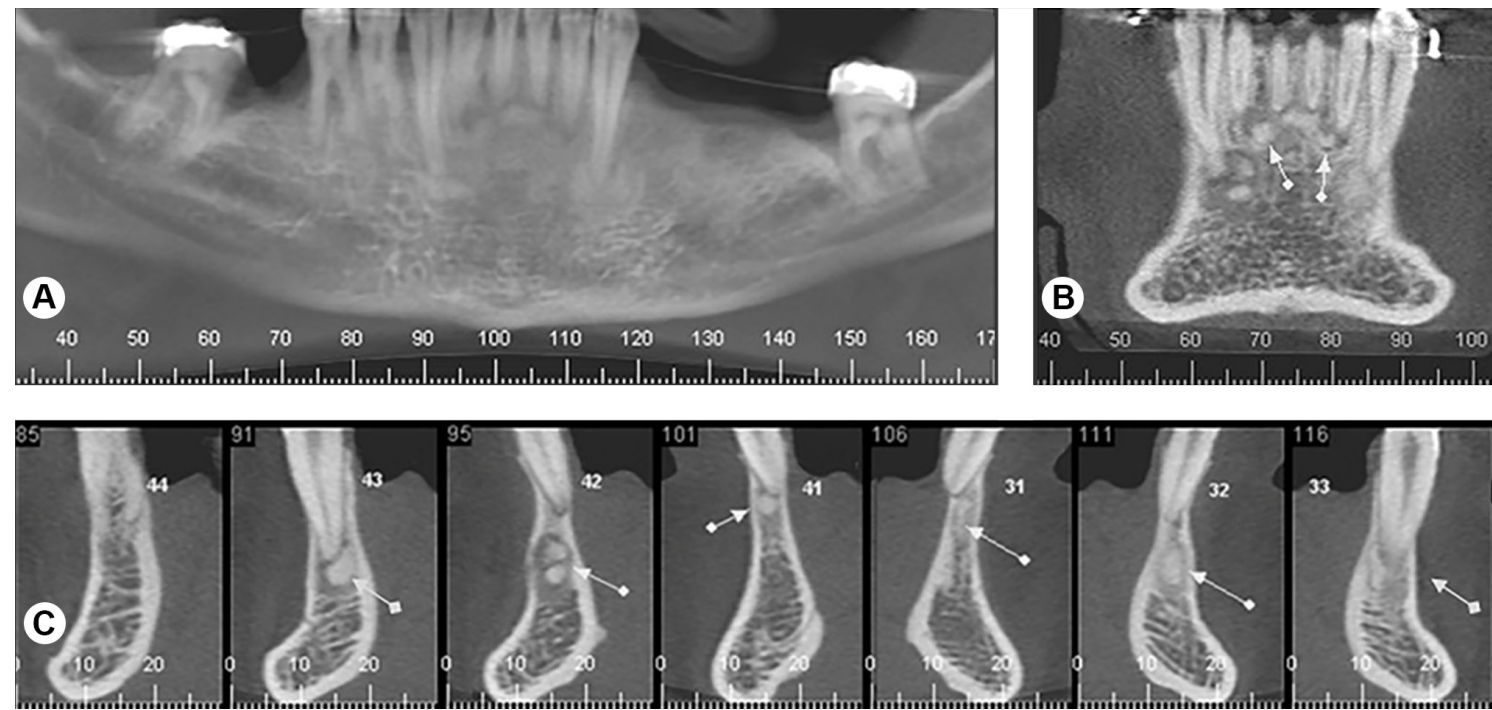

Figure 1. Periapical COD. CBCT images (A, panoramic; B, coronal; and c, cross-sectional reconstructions) showing well-defined, hyperdense lesions, surrounded by hypodense rim, located in the periapex of the mandibular anterior teeth (arrows) and leading to a slight thinning and expansion of the buccal cortical bone in the region of tooth 42 (section 95).
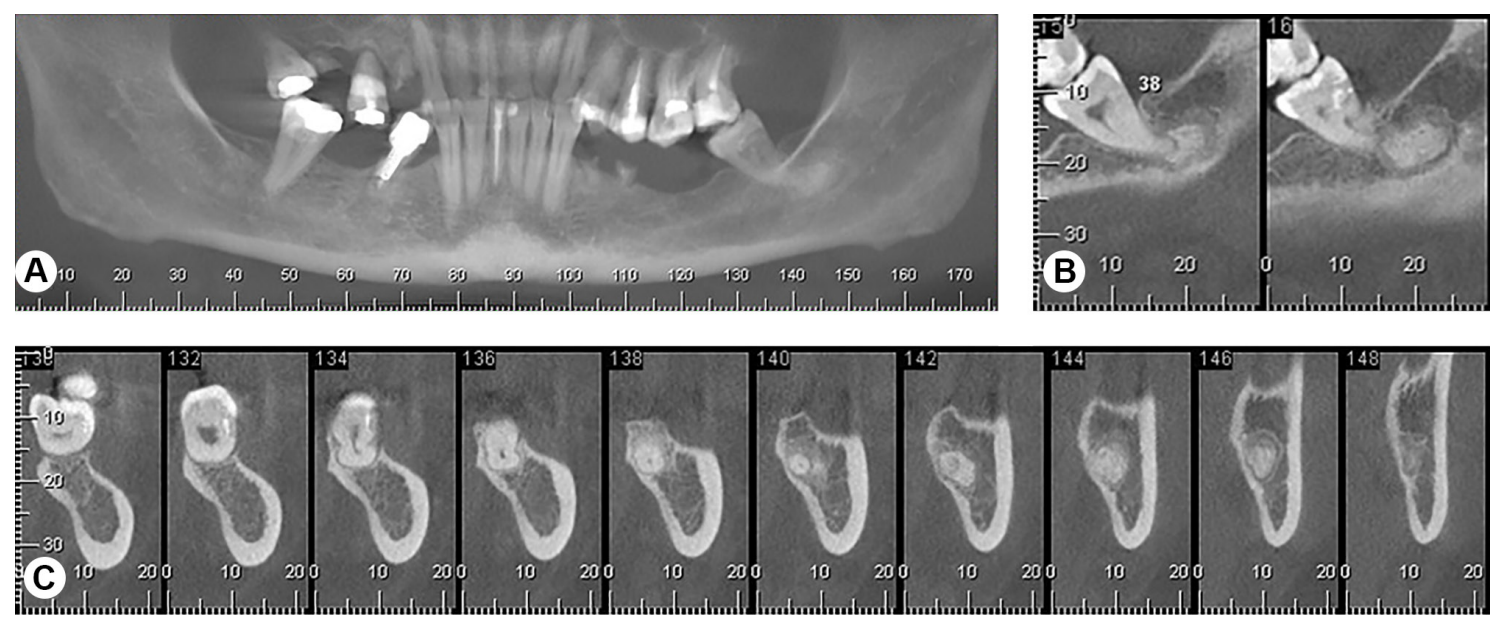

Figure 2. Focal COD. CBCT images (A, panoramic; B, sagittal; and C, cross-sectional reconstructions) demonstrating a well-defined hyperdense image, surrounded by a radiolucent rim, in the periapical region of tooth 38 (sections 140 to 148). 
found in the literature on the contribution of CBCT in the COD evaluation, even with the wide diffusion of this modality of image in several fields of Dentistry. A low prevalence of $\operatorname{COD}(0.4 \%)$ in the general population was observed in this study, in agreement with previous reports (3). However, because of their limited growth and due to being an asymptomatic lesion, their real prevalence is believed to be higher. The actual distribution of each type of fibro-osseous lesion is still unknown, but studies have shown that CODs are the more prevalent type in this group $(1,3,11,13)$. Worawongvasu and Songkampol $(14)$, however, found a very low frequency of COD (0.8-3.3\% florid COD and periapical COD, respectively) among the fibro-osseous lesions. This contradictory result may be explained by the

\section{A Periapical OD}

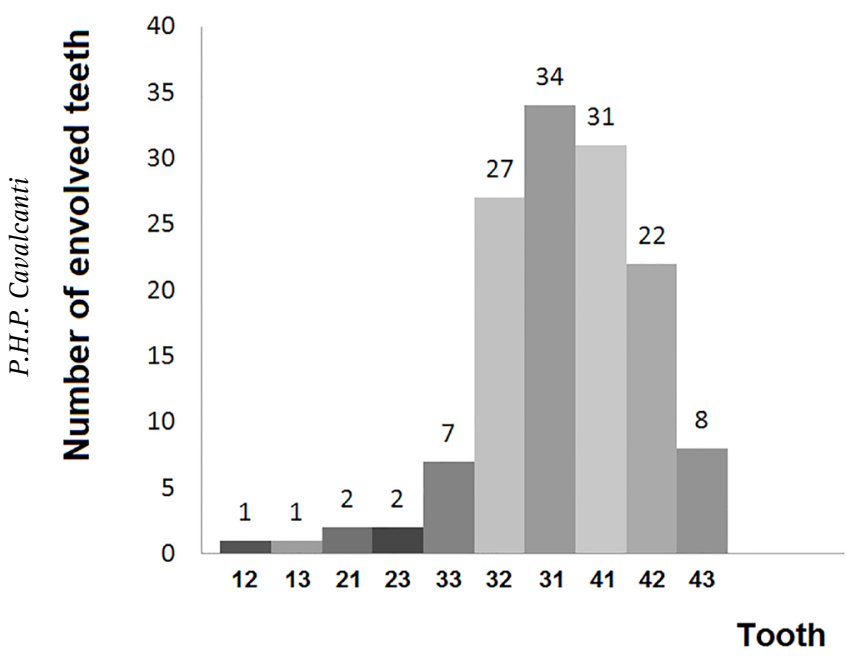

\section{B}

Focal OD

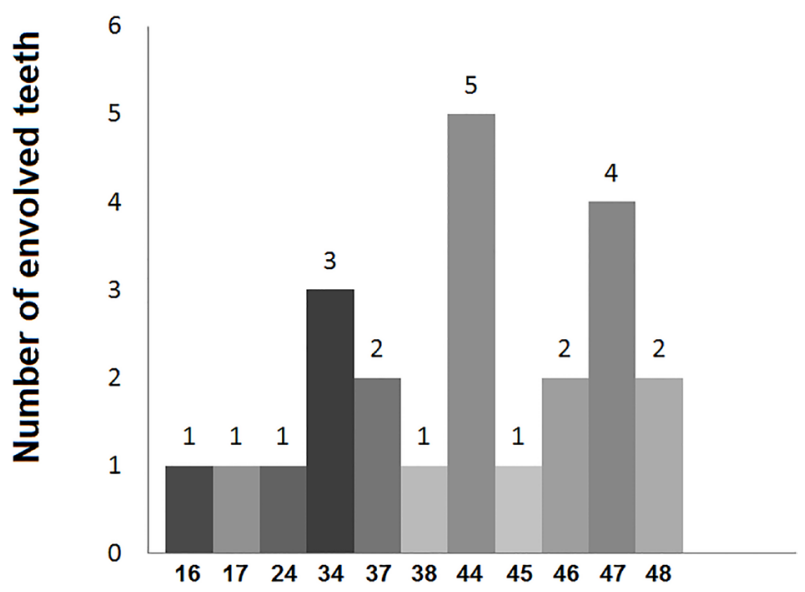

Figure 3. Quantity and distribution of the teeth involved in cases of (A) periapical COD and (B) focal COD. fact that cases in their study required histopathological diagnosis. Nevertheless, biopsies are usually not performed for diagnosis of these lesions. Thus, their prevalence in the study population may have been underestimated.

The data available in literature may vary depending on the population studied $(4,13)$, origin of the cases (pathology laboratories, oral and maxillofacial services), classification used (12) and types of study performed (retrospective studies, clinical follow-up or case reports). In this study, CODs were more common in women, with a mean age between the 5th and 6th decades of life, similar to the findings of other studies $(3,11,12)$. The present study did not include the data on ethnicity of each patient because they were not recorded in the radiological reports, although most studies have found higher predilection for black patients $(3,4,13)$.

Considering the current WHO classification (2), the authors found a higher prevalence of periapical COD $(57.3 \%)$, followed by focal COD $(28 \%)$ and florid COD $(14.6 \%)$. These findings are similar to those of previous reports $(3,12)$ and are in disagreement with the data found by de Noronha Santos Netto et al. (11) who, out of a total of 100 cases of COD, found 65 florid COD, 18 focal COD and only 17 periapical COD. This result may be related to the fact that florid COD is more often symptomatic than the other types of COD, besides their study included only cases with histopathological diagnosis. In agreement with our findings, other studies have also found that the mandible was the bone most involved in $\operatorname{COD}(3,11,12)$. The mandibular central incisors and premolars were the most affected teeth in the periapical and focal COD, respectively. Most florid COD involved only the two quadrants of the mandible, often affecting both the anterior and posterior regions. It is important to note that most lesions evaluated were located near the periapical region of teeth, which could be related to its probable origin from the periodontal ligament. Supporting this hypothesis, Su et al. (3) also noted that $70 \%$ of COD lesions were in close contact with the dental periapex.

Many studies on COD were conducted by evaluating conventional radiographs $(3,6,11,12)$. In this series, we used CBCT images, that allowed all imaging aspects involved in COD to be assessed in detail, without magnification, distortion or overlapping structures, thus representing an advantage in data reliability. In general, COD have welldefined limits and the cases of tooth displacement, root resorption or expansion or rupture of cortical are uncommon $(11,12)$. In the present study, bone cortical expansion was observed in $14.6 \%$ of the cases, distributed on florid and periapical COD. Cases of root resorption and cortical perforation were also identified, mainly associated with the florid COD. These differences can be result in part by the accuracy of CBCT images to detect these details, because 
in many cases, this is not possible with two-dimensional radiographs. According to the literature, florid COD tends to be more expansive, and in a similar study (11), these lesions were observed to be poorly defined in $96 \%$ of cases. These features were not observed in the present study.

The tomographic appearance of COD can vary from hypodense, mixed to hyperdense areas, because over time there is maturation of lesions and deposition of mineralized tissue within them. In this study, many cases of COD presented images with mixed density, similar to other reports $(3,11,12)$. This characteristic presumably occurs both because patients usually seek care during periods of lesion progression, and the clinical and radiological findings of this maturation stage are quite characteristic for its diagnosis. The report presented by Senia and Sarao (5) showed a case of periapical COD in which the lesions progressed through all three stages. They performed a radiographic follow-up for 12 years and observed resolution of lesions, with bone returning to an almost normal trabecular pattern over time. This finding is interesting and shows the importance of a long-term radiographic follow-up for a better characterization of the stages of the disease.

Knowledge of evolution stages of COD is critical for establishing the differential diagnosis with other lesions. Lesions in the initial stage (radiolucent/hypodense) may be misdiagnosed mainly with radicular cysts or periapical granulomas, because of the periapical location. In edentulous areas, periapical OD may be misdiagnosed as residual cysts. Thus, it is of utmost importance to perform a thorough clinical and radiographic evaluation to avoid inadequate procedures, such as endodontic treatments $(1,6,7,9)$, biopsies or even tooth extractions. In the present study, there were 12 teeth affected by COD that were endodontically treated and this could be due to a possible misdiagnosis. In its later stages (mixed/radiopaque density) it may be confused with other fibro-osseous lesions, osteomas or odontogenic tumors (4). Furthermore, the correct diagnosis of radiopaque COD in edentulous areas, differentiating them from bone sclerosis, for example, is essential because of the growing demand for implant placement in these areas, since the presence of COD may contraindicate this procedure (4).

Another possibility of presentation of COD is its association with other diseases, such as simple bone cysts $(12,15,16)$ and osteomyelitis (14). Association between the simple bone cyst and the COD has been found in $12.7 \%$ of cases of COD (15) or 30\% of cases of simple bone cyst (16). These cases usually affect middleaged black women, may be symptomatic and cause cortical expansion $(15,16)$. However, no similar cases were observed in the present study. In addition, CODs have been associated with osteomyelitis in $11 \%$ of cases. Dentoalveolar surgeries, such as extractions and bone biopsy 
in areas affected by COD, are indicated as the main factors for the occurrence of osteomyelitis (14).

This was a retrospective study and there was no possibility of correlation with clinical data of tooth involved, such as pulp sensitivity. Thus, no case of hypodense lesion was diagnosed as COD. While the distinction between a COD during the initial phase and chronic periapical lesions is difficult $(5,7)$, the absence this clinical information may have slightly underestimated the actual prevalence of lesions in this stage. However, this study was valuable because it allowed a data survey from a large initial sample, which would not be possible in a longitudinal clinical or radiographic follow-up study.

Understanding of COD characteristics and the ability to interpret CBCT images are essential for its correct diagnosis. In a study evaluating the accuracy of the diagnosis of COD between radiologists and general dentists, with regard to their pathognomonic characteristics, general dentists were found to be significantly less efficient in the correct interpretation of images (17). This is cause for concern, since these professionals are primarily responsible for the diagnosis and management of these patients. Thus, we emphasize the importance of this study in describing the most common characteristics of COD in CBCT images and discussing them with data in the literature. Dental practitioners should keep in mind the importance of careful imaging evaluation to enable them to recognize these lesions, avoiding misdiagnoses and inadequate treatments.

In conclusion, cemento-osseous dysplasia was more prevalent in female patients with a mean age of 50 years. The periapical COD was the most common type. The mandible was the most affected bone in all types of COD. Most lesions appeared as hyperdense or of mixed density and all had well-defined limits. There were no cases of tooth displacement, but expansion and thinning on cortical plates were relatively common findings, mainly in cases periapical and florid COD. The imaging evaluation and its correlation with clinical and demographic data is critical for their diagnosis.

\section{Resumo}

Os exames por imagem têm papel importante no diagnóstico da displasia cemento-óssea (DCO). A tomografia computadorizada por feixe cônico (TCFC) se destaca por permitir a avaliação tridimensional da imagem. 0 objetivo neste estudo foi avaliar a prevalência de casos diagnosticados como DCO nos exames de TCFC, bem como identificar as principais caracteristicas de imagem relacionadas a essas lesões. Uma análise foi realizada em um banco de dados contendo 22.400 laudos radiológicos, no qual todos os casos que apresentavam algum tipo de DCO foram inicialmente selecionados. Estes exames foram reavaliados para confirmar o diagnóstico radiográfico e determinar a prevalência e distribuição dos tipos de DCO em relação ao sexo, idade e localização preferencial, além de descrever seus aspectos imaginológicos mais comuns. Os dados foram apresentados por meio de análise descritiva. Oitenta e dois casos foram diagnosticados como DCO nas imagens de TCFC (prevalência de 0,4\%).
A distribuição dos pacientes foi de $11(13,4 \%)$ homens e 71 (86,6\%) mulheres, com idade média de 49,8 anos (faixa etária de 17 a 85 anos). Houve 47 (57,3\%) casos de DCO periapical, 23 (28\%) de DCO focal e 12 $(14,6 \%)$ de DCO florida. A mandibula foi mais afetada que a maxila. Na maioria dos casos, as lesões foram mistas ou hiperdensas. Todas as DCO apresentaram limites bem definidos e não houve casos de deslocamento dentário. Em conclusão, a DCO periapical foi o tipo mais comum e o osso mais afetado foi a mandibula. A avaliação da imagem é critica para o seu diagnóstico e os dentistas devem ter em mente todas as possiveis apresentações radiográficas da DCO, a fim de prevenir diagnósticos

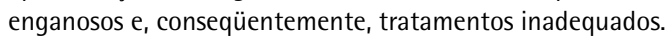

\section{References}

1. Waldron CA. Fibro-osseous lesions of the jaws. J Oral Maxillofac Surg 1993;51:828-835.

2. El-Naggar AK, Chan JKC, Grandis JR, Takata T, Slootweg PJ. WHO classification of head and neck tumours. 4th Ed. Lyon: IARC, 2017.

3. Su L, Weathers DR, Waldron CA. Distinguishing features of focal cemento-osseous dysplasia and cemento-ossifying fibromas. II. A clinical and radiologic spectrum of 316 cases. Oral Surg Oral Med Oral Pathol Oral Radiol Endod 1997;84:540-549.

4. MacDonald-Jankowski DS. Focal cemento-osseous dysplasia: a systematic review. Dentomaxillofac Radiol 2008;37:350-360.

5. Senia ES, Sarao MS. Periapical cemento-osseous dysplasia: a case report with twelve-year follow-up and review of literature. Int Endod J 2015;48:1086-1099.

6. Sirotheau Corrêa Pontes F, Paiva Fonseca F, Souza de Jesus A, Garcia Alves AC, Marques Araújo L, Silva do Nascimento L, et al.. Nonendodontic lesions misdiagnosed as apical periodontitis lesions: series of case reports and review of literature. J Endod 2014;40:16-27.

7. Galgano C, Samson J, Küffer R, Lombardi T. Focal cemento-osseous dysplasia involving a mandibular lateral incisor. Int Endod J 2003;36:907-911.

8. Ortega A, Fariña V, Gallardo A, Espinoza I, Acosta S. Nonendodontic periapical lesions: a retrospective study in Chile. Int Endod J 2007;40:386-390.

9. Huh JK, Shin SJ. Misdiagnosis of florid cemento-osseous dysplasia leading to unnecessary root canal treatment: a case report. Restor Dent Endod 2013;38:160-166.

10. Sarmento DJ, Monteiro BV, de Medeiros AM, da Silveira EJ. Severe florid cemento-osseous dysplasia: a case report treated conservatively and literature review. Oral Maxillofac Surg 2013;17:43-46.

11. de Noronha Santos Netto J, Machado Cerri J, Miranda AM, Pires FR. Benign fibro-osseous lesions: clinicopathologic features from 143 cases diagnosed in an oral diagnosis setting. Oral Surg Oral Med Oral Pathol Oral Radiol 2013;115:e56-e65.

12. Alsufyani NA, Lam EW. Osseous (cemento-osseous) dysplasia of the jaws: clinical and radiographic analysis. J Can Dent Assoc 2011;77:b70.

13. Eversole R, Su L, El-Mofty S. Benign fibro-osseous lesions of the craniofacial complex: a review. Head Neck Pathol 2008;2:177-202.

14. Worawongvasu R, Songkampol K. Fibro-osseous lesions of the jaws: an analysis of 122 cases in Thailand. J Oral Pathol Med 2010;39:703-708.

15. Chadwick JW, Alsufyani NA, Lam EW. Clinical and radiographic features of solitary and cemento-osseous dysplasia-associated simple bone cysts. Dentomaxillofac Radiol 2011;40:230-235.

16. Peacock ME, Krishna R, Gustin JW, Stevens MR, Arce RM, Abdelsayed RA. Retrospective study on idiopathic bone cavity and its association with cementoosseous dysplasia. Oral Surg Oral Med Oral Pathol Oral Radiol 2015;119:e246-e251.

17. Alsufyani NA, Lam EW. Cemento-osseous dysplasia of the jaw bones: key radiographic features. Dentomaxillofac Radiol 2011;40:141-146. 